\title{
Chorea with acanthocytosis: are we under diagnosing neuroacanthocytosis?
}

\author{
Harshana Samarasinghe ${ }^{1}$, Kamani Liyanarachchi ${ }^{1}$, Saman B Gunatilake ${ }^{1}$ \\ Journal of the Ceylon College of Physicians, 2011, 42, 41-42
}

\begin{abstract}
Neuroacanthocytosis is a genetically heterogenous disorder characterized by neurological abnormalities which can manifest later in life. More than 5\% acanthocytes in a peripheral blood film is supportive of neuroacanthocytosis. Here we report two female patients aged 48 and 33 years who presented with chorea and the possibility of neuroacanthocytosis was not considered initially leading to considerable delay in diagnosis. Since the time of appearance of acanthocytosis among patients vary in the course of the disease, it is necessary to have regular follow up with repeated blood film examination to recognize neuroacanthocytosis in otherwise undiagnosed patients.
\end{abstract}

\section{Introduction}

Neuroacanthocytosis (NA) was first described by Bassen and Kornzweig in 1950. It was a case of inherited abetalipoproteinaemia associated with acanthocytosis and ataxia. The second type of neuroacanthocytosis was described in 1960 by Levine and in 1968 by Critchley. Levine-Critchley syndrome differs from Huntington disease (HD) by the presence of acanthocytosis and from Bassen-Kornzweig disease by normal lipoproteins as well as a later age of onset. After that there were many reported case with or without associated abetalipoproteinaemia.

Neuroacanthocytosis describes a group of genetically heterogenous disorders characterized by neurological abnormalities associated with acanthocytosis. Neurologic problems usually manifest as, either movement disorders or ataxia, personality changes, cognitive deterioration, axonal neuropathy, and seizures. Most patients manifest acanthocytosis on the peripheral blood smear at some point during the course of the disease ${ }^{1,2}$. Usually $10-30 \%$ of the patients' erythrocytes have an unusual star-like appearance with spiky- or thorny-appearing projections, which are acanthocytes. Here we report two cases of NA.

\footnotetext{
${ }^{1}$ Colombo South Teaching Hospital.
}

\section{Case 1}

Patient was a 48-year old female who presented with progressive worsening choreiform movements of four years duration. On presentation she had abnormal movements including chorea, tics and orofacial dyskinesia. She also had aggressive behaviour and poor self care. She was a product of a non consanguineous marriage. One of her elder brothers of her ten siblings had abnormal movements similar to hers. He had not been investigated to date. She was not on any long term drugs before her symptoms started. Her neurological examination was normal except for her abnormal movements. Her fresh blood film revealed $20-30 \%$ of acanthocytes. CT brain showed cerebral atrophy. She had a high indirect bilirubin level. Other liver function tests were normal. Kayser-Fleischer rings were not seen. Her serum copper and ceruloplasmin and $24 \mathrm{hr}$ urinary copper excretion were in the normal range. There was no polycythemia, no history or echo cardiographic evidence of rheumatic carditis. Her thyroid functions were normal, ANA negative and her lipid profile was normal.

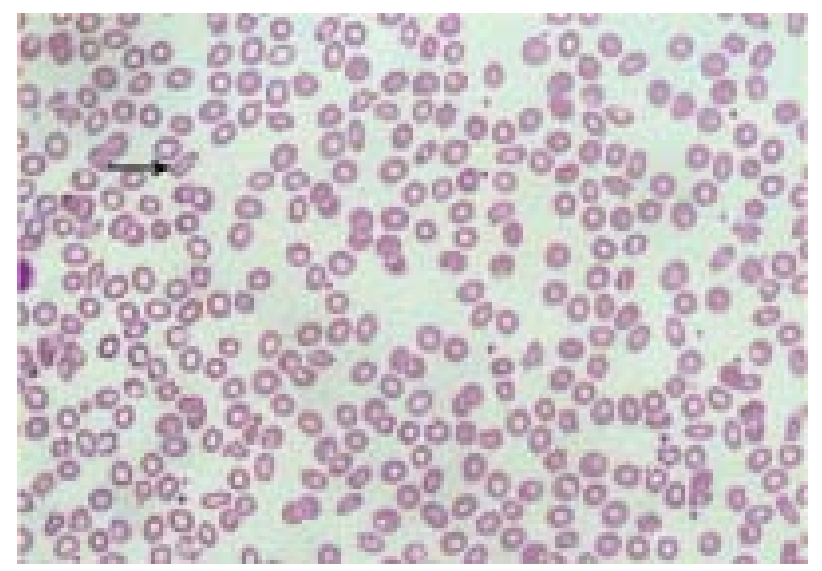

Figure 1

\section{Case 2}

This 33-year old female who had progressively worsening chorea involving both upper limbs and lower limbs and head recently had developed ataxia and swallowing difficulty. She was a product of non consanguineous marriage with no family history of movement disorders. Higher functions and cranial 
nerves were normal. Her upper limbs' and lower limbs' tone, power and reflexes were normal. There was no sensory impairment. Eye examination didn't show Kayser-Fleischer rings. Her SGOT liver enzymes were normal, ANA was negative. MRI brain and ultra sound scan abdomen were normal. Her lipid profile was normal. Genetic study had excluded Huntington disease. Hb was $11.1 \mathrm{~g} / \mathrm{dl}$ with normochromic normocytic red cells and a fresh blood film examined without adding EDTA showed over $60 \%$ of acanthocytes.

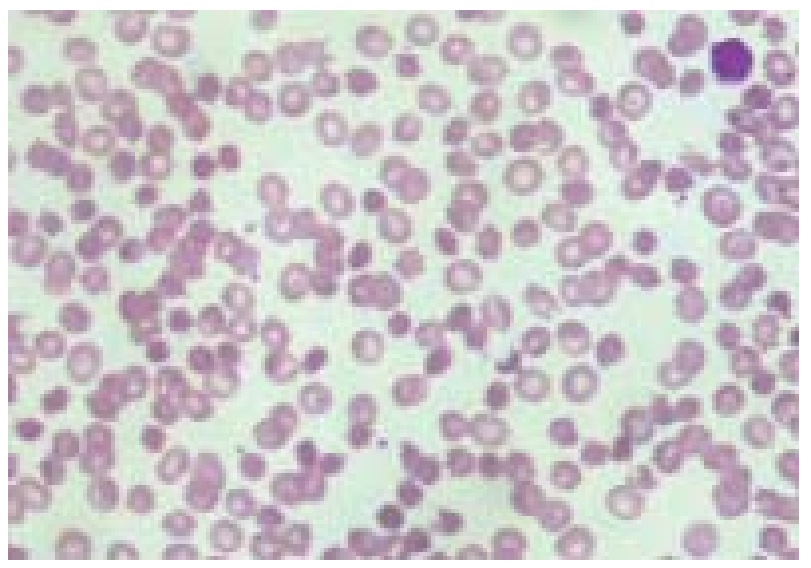

Figure 2

\section{Discussion}

Neuroacanthocytosis causes a movement disorder which closely resembles Huntington's chorea. However, it is not usually considered in the aetiology of patients presenting with chorea. Both our patients were investigated prior to this presentation for several years and the most of the secondary causes of chorea were excluded without entertaining the possibility of neuroacanthocytosis. Neuroacanthocytosis was suspected with the presence of orofacial and lingual dyskinesias, early age at onset and absence of an autosomal dominant inheritance in the family history. In one patient genetic studies were negative for HD mutation. In addition dysphagia, seizures, areflexia, axonal neuropathy and myopathy also should raise suspicion of neuroacanthocytosis.

Neuroacanthocytosis can be reliably diagnosed with the clinical history and the investigations. Demonstration of a significant acanthocytosis (>5\%) in a peripheral blood film strongly supports the diagnosis of neuroacanthocytosis. Both our patients showed a marked acanthocytosis. The examination of fresh blood without EDTA is necessary for acanthocyte detection and to avoid false positives caused by common artefacts or echinocytes, which may be present normally in small numbers ${ }^{3}$. Normal lipoproteins in both patients excluded abetalipoproteinemia (Bassen-Kornzweig syndrome). Arriving at a specific diagnosis is important to understand the gene implications and to offer appropriate genetic counseling for the patients although it may not significantly change the management. Examination of a fresh blood film for acanthocytes should be an integral part in the assessment of a patient presenting with progressive choreiform disorder.

\section{References}

1. Sorrentino G, De Renzo A, Miniello S, Nori O, Bonvita V. Late appearance of acanthocytes during the course of choreaacanthocytosis. Journal of the Neurological Sciences 1999; 163: $175-8$.

2. Bayreuther C, Borg M, Ferrero-Vacher C, Chaussenot A, Lebrun C. Revue Neurologique (Paris) 2010; 166: 100-3.

3. Kanjanasut N, Jagota P, Bhidayasiri R. The first case report of neuroacanthocytosis in Thailand: utilization of a peripheral blood smear technique for detecting acanthocytes. Clinical Neurology and Neurosurgery 2010; 112: 541-3. 